[0212-7199 (2005) 22: 5; pp 222-226] ANALES DE MEDICINA INTERNA Copyright $(2005$ ARAN EDICIONES, S.L.

An. Med. INTERNA (Madrid) Vol. 22, N. ${ }^{\circ} 5$, pp. 222-226, 2005

\title{
Estudio epidemiológico de la tuberculosis en un hospital de tercer nivel en el año 2001
}

\author{
I. GARCÍA SÁNCHEZ, C. PÉREZ DE OTEYZA, C. GILSANZ FERNÁNDEZ \\ Servicio de Medicina Interna I. Hospital General Universitario Gregorio Marañón.
} Madrid

\author{
TUBERCULOSIS EPIDEMIOLOGICAL STUDY IN A THIRD LEVEL \\ HOSPITAL DURING 2001
}

\section{RESUMEN}

Objetivos: Estimar la incidencia y características clínicoepidemiológicas de la Tuberculosis (TB), analizando la influencia de factores predisponentes como la infección VIH, la inmigración, enfermedades crónicas y hábitos de vida.

Material y métodos: Se realizo un estudio retrospectivo de los pacientes diagnosticados de TB activa (confirmación microbiológica) y latente (presencia de Mantoux positivo) durante el año 2001, en el hospital Gregorio Marañón. Las variables incluidas se referían a datos epidemiológicos, bacteriológicos, clínicos y terapéuticos.

Resultados: Se detectaron 78 pacientes con TB en hospital, con una incidencia estimada en el área de salud de 11,14 casos por 100.000 habitantes. La edad media fue de 41,98 años; con 43,6\% pacientes VIH positivos y $9 \%$ inmigrantes. La muestra microbiológica más utilizada fue el esputo, detectándose resistencias en $30 \%$ de los casos donde se realizo. De los 78 casos revisados, 64 (82\%) tenían TB activa. En los pacientes VIH fue más frecuente la TB por reactivación $(p=0,016)$. La localización predominante fue la pulmonar $(78 \%)$. El hallazgo radiológico más frecuente fue un infiltrado pulmonar. El $56 \%$ de los pacientes VIH presentaron radiografías normales $(\mathrm{p}<0,05)$. El tratamiento más habitual fue con cuatro fármacos y el $75 \%$ de los pacientes lo cumplieron correctamente; el abandono fue más frecuente en los pacientes VIH positivos ( $\mathrm{p}>0,01)$. La mortalidad global fue del $9 \%$.

Conclusiones: En la actualidad, en Madrid, la población con mayor riesgo de TB son los sujetos con infección VIH, drogadicción, inmigrantes y ancianos institucionalizados. Para un adecuado control de la enfermedad es necesario un diagnóstico precoz y una supervisión del tratamiento.

PALABRAS CLAVE: Tuberculosis. Infección VIH. Inmigración. Factores epidemiológicos.

\begin{abstract}
Objectives: Estimate the incidence and epidemiological and clinical characteristics of Tuberculosis (TB), analyzing the influence of factors such as: HIV infection, immigration, chronic diseases and styles of life.

Material and methods: Retrospective study of patients who have diagnosed of active TB (microbiological results positive) and latent tuberculosis (Mantoux positive) during the year 2001 in the Gregorio Marañon Hospital. The variables includes epidemiological, microbiological, clinicals and therapeutics features.

Results: We were registered 78 patients with $T B$ at hospital, with estimated incidence in health area of 11,14 cases per 100.000 habitants. The mean age of were 41,98 years; with 43,6\% HIV infected patients and $9 \%$ immigrants. The sputum was the sample most used for the microbiological diagnosis, we detected resistance in $30 \%$ of samples analysed. The HIV infected patients had more frequent TB from reactivation $(p=0,016)$. The primary site of disease was pulmonary (78\%). One pulmonary infiltrate was more frequent in the thoracic X-ray. The $56 \%$ of our HIV infected patients had normal thoracic $X$-ray $(p<0,05)$. The treatment more common was with four drugs and the $75 \%$ of our patients fulfilled the treatment; the HIV infected patients leaves the treatment more frequent. The global mortality was $9 \%$.

Conclusions: Actually, in Madrid, the persons with HIV infection, injection drug abuse, immigration and elders in residential home were higher risk of infection for TB. For strict control of disease is need early diagnosis and supervision of treatment .
\end{abstract}

KEY WORDS: Tuberculosis. HIV infection. Immigration. Epidemiological factors.

García Sánchez I, Pérez de Oteyza C, Gilsanz Fernández C. Estudio epidemiológico de la tuberculosis en un hospital de tercer nivel en el año 2001. An Med Interna (Madrid) 2005; 22: 222-226.

\section{INTRODUCCIÓN}

La tuberculosis (TB) constituye, en el momento actual, un grave problema de salud pública a escala mundial. En este sentido, la Organización Mundial de la Salud (OMS) desde 1993, la considera una emergencia medica, estimándose que más de un tercio de la población mundial esta infectada por el bacilo Mycobacterium tuberculosis; con 8 millones de casos nuevos y dos millones de muertes cada año, calculándose para el año 2000, una incidencia de 10,2 millones de casos de TB en todo el planeta (1-3).

El 90\% de los casos de TB se concentran en áreas subdesarrolladas; pero los países desarrollados no están libres de este problema sanitario, aunque la situación de la epidemia es 
diferente según el país considerado. En los últimos años se ha producido recrudecimiento de la enfermedad; debido, la extensión del sida, la inmigración y el aumento de ancianos institucionalizados, a menudo con enfermedades crónicas que favorecen la reactivación de procesos antiguos (4).

Por otra parte, en España la TB es la principal infección oportunista en los pacientes infectados por el VIH (5). La enfermedad tuberculosa en los individuos VIH se suele presentar de forma atípica; es frecuente el incumplimiento terapéutico, favoreciendo la reactivación de la enfermedad y la aparición de resistencias (6).

Ante estos datos decidimos realizar un estudio para estimar la incidencia y las características sociales, clínicas y terapéuticas de la TB en un hospital terciario de Madrid; analizando la influencia de factores como la infección VIH, la inmigración, enfermedades crónicas y los hábitos de vida predisponentes para la TB.

\section{MATERIAL Y MÉTODOS}

Se ha realizado un estudio descriptivo, retrospectivo, de los pacientes diagnosticados de TB durante el año 2001 (enero a diciembre) en el Hospital General Universitario Gregorio Marañón. Dicho centro es el hospital de referencia del área sanitaria 1 de Madrid, que atiende a una población estimada de 700.000 habitantes, con nivel socioeconómico medio-bajo.

La TB se clasificó como TB activa, en los pacientes con resultado positivo del BAAR y/o cultivo en medio de Löwenstein-Jensen; y latente en los que presentaban un Mantoux positivo (se considera resultado positivo $5 \mathrm{~mm}$ de induración sí existe baja respuesta inmune o reciente contacto con un paciente bacilífero, $10 \mathrm{~mm}$ en individuos con mayor riesgo de infección y de $15 \mathrm{~mm}$ en el resto de la población) (7). En los casos de TB activa, diferenciamos entre los casos iniciales y aquellos debidos a reactivación de un proceso antiguo por recaída de la enfermedad o abandono del tratamiento.

Las variables del estudio fueron:

1. Sociales: edad, sexo.

2. Factores de riesgo: hábitos tóxicos como la adicción a drogas vía parenteral (ADVP) o alcoholismo; enfermedades crónicas: diabetes mellitus, insuficiencia renal, neoplasias, uso de fármacos inmunosupresores; y situaciones como estancia en prisión, inmigrante, vagabundo o contacto con enfermos bacilíferos.

3. Bacteriológicas: tipo de muestra, baciloscopia y/o cultivo; estudio de resistencias a fármacos tuberculostasticos; prueba de la tuberculina (administración de 2 unidades de tuberculina PPD CT-68 por vía intradérmica en la cara anterior del brazo, realizándose la lectura a las 72 horas, midiendo los centímetros de induración según el diámetro transversal al eje longitudinal del antebrazo).

4. Clínicas: localización de la enfermedad; presencia de síntomas respiratorios, síndrome constitucional, o fiebre; y resultados de la radiografía de tórax.

5. Terapéuticas: pauta de tratamiento utilizada, duración y cumplimiento terapéutico.

Se ha utilizado el programa estadístico SPSS. Las variables cuantitativas se expresaron con la media y desviación estándar (SD). Para la comparación entre las distintas variables cuantitativas se utilizo la prueba de la t de Student y para las variables cualitativas la prueba Chi-cuadrado y exacta de
Fischer. Se considero que las diferencias eran estadísticamente significativas cuando $\mathrm{p}<0,05$.

\section{RESULTADOS}

Se registraron 78 pacientes con diagnóstico de TB, entre 13.601 adultos ingresados en los servicios médicos del hospital en el año 2001; con una incidencia del 0,5\% de la TB en el hospital. En el área 1 de salud, la incidencia estimada sería de 11,14 casos por 100.000 habitantes.

En el conjunto de la muestra la edad media fue de 41,98 años $(\mathrm{SD}=18,41)$; en la distribución por sexo predominan los varones $(77 \%)$. Se detectó coinfección VIH en 34 pacientes $(43,6 \%)$. En la tabla I, podemos observar las características epidemiológicas según la presencia de coinfección VIH, destacando que los pacientes VIH positivos se diferencian de los VIH negativos, de forma estadísticamente significativa en una menor edad media $(37,02$ años, $\mathrm{SD}=22,88)$ y mayor porcentaje de adicción a drogas por vía parenteral (85\% ADVP). Por el contrario, entre los pacientes VIH negativos, hay mayor proporción de alcohólicos y de diabéticos, aunque las diferencias no fueron significativas.

\section{TABLA I}

CARACTERÍSTICAS EPIDEMIOLÓGICAS DE LA POBLACIÓN DEL ESTUDIO

\begin{tabular}{lcccc}
\hline & $\begin{array}{c}\text { Pob. total } \\
n=78\end{array}$ & $\begin{array}{c}\text { VIH } \\
n=34\end{array}$ & $\begin{array}{c}\text { No VIH } \\
n=44\end{array}$ & $\begin{array}{c}\text { Diferencias } \\
(p)\end{array}$ \\
\hline Varón & $60(76,9 \%)$ & $29(48,3 \%)$ & $31(70,5 \%)$ & 0,123 \\
Mujer & $18(23,1 \%)$ & $5(14,7 \%)$ & $13(29,5 \%)$ & 0,123 \\
Edad & 41,98 & 37,02 & 45,81 & 0,021 \\
& $(S D=18,41)$ & $(S D=8,01)$ & $(S D=22,88)$ & \\
ADVP* & $34(44 \%)$ & $29(85,3 \%)$ & $5(11,4 \%)$ & $<0,001$ \\
Alcohólicos & $23(29 \%)$ & $8(23,5 \%)$ & $15(34 \%)$ & 0,30 \\
Diabetes mellitus & $7(9 \%)$ & $1(2,9 \%)$ & $6(13,6 \%)$ & 0,07 \\
Insuficiencia renal & & & & \\
crónica & $1(1,3 \%)$ & $1(2,9 \%)$ & 0 & 0,31 \\
Neoplasias & $1(1,3 \%)$ & 0 & $1(2,3 \%)$ & 0,31 \\
Tto. CC** & $2(2,6 \%)$ & 0 & $2(4,5 \%)$ & 0,14 \\
Prisión & $5(6,4 \%)$ & $3(8,8 \%)$ & $4(9,1 \%)$ & 0,96 \\
Vagabundo & $10(12,8 \%)$ & $6(17,6 \%)$ & $4(9 \%)$ & 0,27 \\
\hline
\end{tabular}

(*) Adictos a drogas por vía parenteral.

${ }^{* *}$ ) Tratamiento crónico con corticoides.

La figura 1, que representa la distribución por edad de la población, muestra un máximo de edad entre el periodo de 2040 años, representado principalmente por pacientes VIH.

Con respecto al país de origen, 7 de los 78 de los pacientes (9\%) eran inmigrantes; 4 de estos procedían de países hispanoamericanos, 2 de países africanos y uno Europa del Este. La mayoría de los inmigrantes eran varones con un $86 \%$; no existían diferencias significativas con la población española del estudio $(\mathrm{p}=0,91)$. La edad media de los inmigrantes era de 28,57 años ( $\mathrm{SD}=12,10)$; con diferencias estadísticamente significativas $(\mathrm{p}=0,017)$, al comparar con la edad media de los pacientes no inmigrantes. Respecto a su estancia en España, la mayoría de los inmigrantes (57\%) del estudio llevaban más de un año en nuestro país y el resto menos de tres meses. 


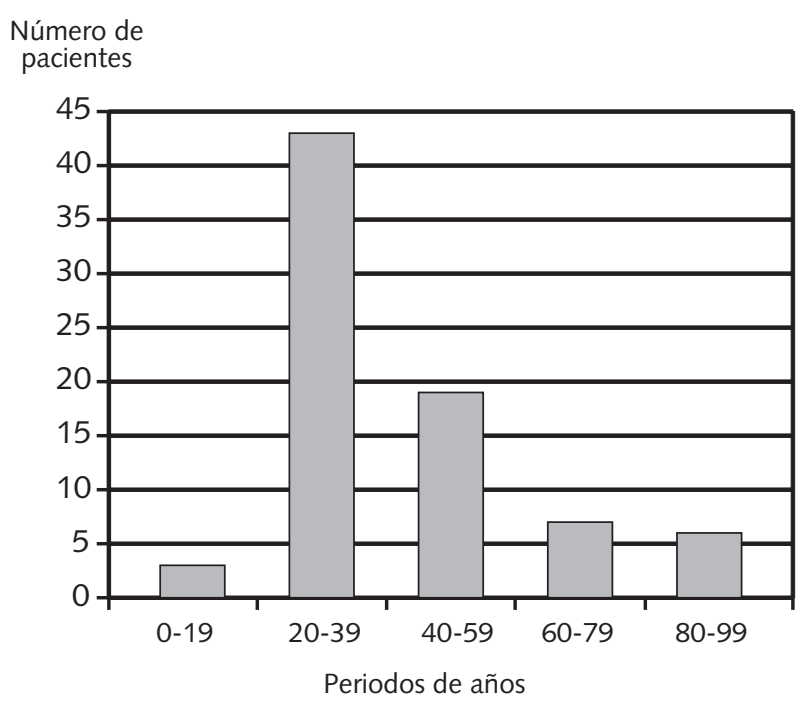

Fig. 1. Distribución de la tuberculosis según la edad en 78 casos.

En la tabla II, se observa los procedimientos microbiológicos utilizados en el estudio: la muestra más utilizada para el diagnóstico fue el esputo, con un $73 \%$ de resultados positivos en la tinción de BAAR y un $51 \%$ de cultivos positivos para $M$. tuberculosis; en el $40 \%$ de las muestras fueron positivas ambas técnicas. Al comparar el rendimiento de dichas pruebas según la presencia de coinfección VIH: se aprecia una mayor proporción de cultivos de esputo positivos (22\%) entre los pacientes VIH positivos; aunque el resultado positivo del BAAR y cultivo de esputo (53\%) fue mayor en los VIH negativos, de forma estadísticamente significativa. En el $21 \%$ de los pacientes, el diagnostico microbiológico se realizo en otras muestras.

La prueba de la tuberculina (PPD) se realizó en 34 pacientes $(43,6 \%)$ del estudio, con un resultado positivo en $26(76 \%)$ de ellos. Dicha prueba se realizo en 15 pacientes VIH negativos presentaron con un $93 \%$ resultados positivos, frente al un $63 \%$ de los 19 pacientes VIH positivos donde se realizo el test, diferencias estadísticamente significativas $(p=0,018)$.

\begin{tabular}{|c|c|c|c|c|}
\hline \multicolumn{5}{|c|}{ TABLA II } \\
\hline \multicolumn{5}{|c|}{$\begin{array}{l}\text { RESULTADOS DE LAS MUESTRAS MICROBIOLÓGICAS DE TB } \\
\text { ACTIVA }\end{array}$} \\
\hline $\begin{array}{l}\text { Muestras } \\
\text { positivas }\end{array}$ & $\begin{array}{c}\text { Pob. total } \\
n=64\end{array}$ & $\begin{array}{c}\text { VIH } \\
n=23\end{array}$ & $\begin{array}{c}\text { No VIH } \\
n=41\end{array}$ & $\begin{array}{c}\text { Diferencias } \\
(p)\end{array}$ \\
\hline BAAR en esputo & $21(32 \%)$ & $9(39 \%)$ & $12(29 \%)$ & 0,42 \\
\hline Cultivo de esputo & $7(11 \%)$ & $5(22 \%)$ & $2(5 \%)$ & 0,067 \\
\hline \multicolumn{5}{|l|}{ BAAR y cultivo } \\
\hline de esputo & $26(40 \%)$ & $3(13 \%)$ & $22(53 \%)$ & $<0,001$ \\
\hline Otras muestras & $14(21 \%)$ & $7(30 \%)$ & $6(14 \%)$ & 0,15 \\
\hline Hemocultivos & $5(0,08)$ & $4(17 \%)$ & $1(24 \%)$ & 0,07 \\
\hline Adenopatías & $2(3 \%)$ & $2(8 \%)$ & 0 & 0,13 \\
\hline Biopsia pleural & $3(4 \%)$ & 0 & $3(7 \%)$ & 0,071 \\
\hline Biopsia articular & $2(3 \%)$ & 0 & $2(5 \%)$ & 0,14 \\
\hline Coprocultivo & $1(1 \%)$ & 0 & $1(2 \%)$ & 0,31 \\
\hline LCR & $1(1 \%)$ & $1(4 \%)$ & 0 & 0,30 \\
\hline
\end{tabular}

El análisis de resistencias se realizó en 20 muestras de las 64 utilizadas en el estudio y se detectaron dichas resistencias en 6 muestras (30\%). Las características de los casos resistentes se resumen en la tabla III.

\section{TABLA III}

CARACTERÍSTICAS DE LOS CASOS DE TB RESISTENTE

\begin{tabular}{|c|c|c|c|c|c|c|}
\hline $\mathrm{N}^{0}$ de caso & 1 & 2 & 3 & 4 & 5 & 6 \\
\hline Edad & 32 & 80 & 26 & 24 & 54 & 29 \\
\hline Sexo & Varón & Varón & Varón & Varón & Varón & Mujer \\
\hline VIH & No & No & Sí & Sí & No & No \\
\hline CD4 & & & $<200$ & $<200$ & & \\
\hline Localización & Pulmón & Pulmón & Extra* & Pulmón & $P+$ extra** & P+extra \\
\hline Resistencias & $R, S, I$ & PIR & $R$ & 1 & $\mathrm{I}, \mathrm{ET}$ & $S$ \\
\hline Tipo & Primaria & Primaria & Secund. ** & Secund. & Primaria & Primaria \\
\hline Tratamiento & $2^{\mathrm{a}}$ línea & $I, R, E$ & No & $R, E, P$ & $2^{\mathrm{a}}$ línea & $\mathrm{I}, \mathrm{R}, \mathrm{P}$ \\
\hline Evolución & Buena & Buena & Mala & Buena & Cirugía & Buena \\
\hline
\end{tabular}

R: Rifampicina; S: estreptomicina; I: Isoniazida; ET: Etambuntol; PIR: Pirazinamida. $2^{a}$ línea: tratamiento con fármacos de segunda línea. $\left({ }^{*}\right)$ : localización extrapulmonar. $\left.{ }^{* *}\right)$ : localización pulmonar más extrapulmonar. $(* *)$ : resistencias.

Respecto a las formas clínicas de las TB estudiadas, 64 pacientes (82\%) tenían enfermedad activa y $14(17 \%)$ eran formas latentes. De los casos de TB activa, 40 (62\%) eran formas iniciales, mientras que $24(36 \%)$ eran reactivaciones: 12 por abandono terapéutico y los otros 12 por fracaso del tratamiento.

De los casos de enfermedad activa 23 se presentaron en los pacientes con coinfección VIH, con 10 formas iniciales (43\%) y 30 formas iniciales en 41 pacientes VIH negativos con TB activa. Mientras que la reactivación de la enfermedad, se presento en $13(56 \%)$ pacientes VIH positivos y en 11 (26\%) VIH negativos, las diferencias fueron estadísticamente significativas $(\mathrm{p}=0,016)$.

En la figura 2, que representa la localización de la enfermedad, se observa que predominan los casos de TB pulmonar.

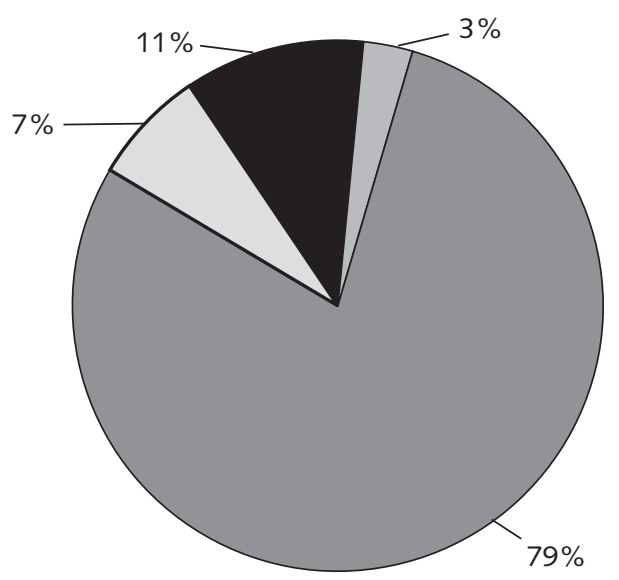

Pulmonar $\square$ Diseminad. $\square$ P. + extrap. $\square$ Extrap.

Fig. 2. Distribución de los casos de tuberculosis activa según su localización anatómica. 
TABLA IV

CLÍNICA Y RADIOLOGÍA DE LOS CASOS DE TB ACTIVA

\begin{tabular}{lcccc}
\hline & $\begin{array}{c}\text { Pob. total } \\
n=64\end{array}$ & $\begin{array}{c}\text { VIH } \\
n=23\end{array}$ & $\begin{array}{c}\text { No VIH } \\
n=41\end{array}$ & $\begin{array}{c}\text { Diferencias } \\
(p)\end{array}$ \\
\hline Síntomas respiratorios & $50(78 \%)$ & $20(87 \%)$ & $30(73 \%)$ & 0,16 \\
Fiebre & $32(50 \%)$ & $15(65 \%)$ & $17(41 \%)$ & 0,058 \\
Síndrome & & & & \\
$\quad$ constitucional & $35(54 \%)$ & $13(56 \%)$ & $22(54 \%)$ & 0,82 \\
Infiltrado pulmonar & $25(39 \%)$ & $13(56 \%)$ & $12(29 \%)$ & 0.029 \\
Caverna tuberculosa & $20(30 \%)$ & $3(13 \%)$ & $17(41 \%)$ & 0,006 \\
Derrame pleural & $3 / 64(4 \%)$ & 0 & $3 / 41(7 \%)$ & 0,071 \\
Pérdida de volumen & $7(10 \%)$ & $2(8 \%)$ & $5(12 \%)$ & 0,65 \\
Adenopatías & $5(7 \%)$ & $3(13 \%)$ & $2(5 \%)$ & 0,29 \\
Rx. Normal & $18(28 \%)$ & $13(56 \%)$ & $5(12 \%)$ & 0,0001 \\
\hline
\end{tabular}

En la tabla IV, se presentan las manifestaciones clínicas y datos radiológicos, así como con las diferencias entre los pacientes VIH positivos y negativos. No hubo diferencias significativas en las manifestaciones clínicas. La presencia de un infiltrado pulmonar en la radiografía de tórax fue el hallazgo más frecuente en la población del estudio. Pero la radiografía fue normal en el 56\% de los pacientes VIH y no hubo ningún derrame pleural en estos pacientes. Por el contrario, entre los VIH negativos hubo un $41 \%$ de casos con cavitación pulmonar; estas diferencias fueron estadísticamente significativas $(\mathrm{p}<0,05)$.

Respecto las pautas terapéuticas, reflejadas en la figura 3, la más utilizada en un $37 \%$ de los casos de TB activa, fue isoniazida, rifampicina, pirazinamida y etambutol, durante dos meses, y seguida de isoniazida y rifampicina durante cuatro meses más.

En los casos de TB latente, los tratamientos más utilizados fueron: isoniazida durante 9 meses $(57 \%)$, o bien isoniazida, pirazinamida y rifampicina durante tres meses (43\%).

El $75 \%$ de los pacientes incluidos en el estudio cumplieron correctamente el tratamiento prescrito. Entre la población VIH hubo un $26 \%$ de abandonos terapéuticos, frente al $4 \%$ entre los pacientes VIH negativos, siendo las diferencias altamente significativas $(\mathrm{p}<0,01)$.

La mortalidad del estudio fue del $10 \%$, aunque un $50 \%$ fallecieron por causas ajenas a la TB. La mortalidad fue mayor

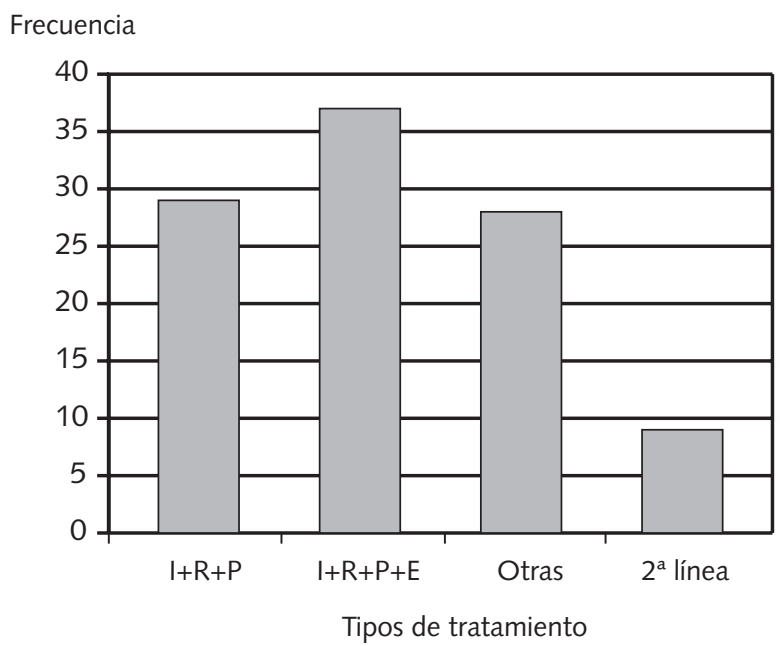

Fig. 3. Pautas de tratamiento en los casos de tuberculosis activa.
$(16 \%)$ entre los pacientes VIH negativos que entre los VIH positivos $(0,03 \%)$, aunque la diferencia no sea significativa.

\section{DISCUSIÓN}

España es el segundo país de la Unión Europea, después de Portugal, con mayor número de casos de TB. En concreto cada año se detectan 12.000 casos nuevos. La incidencia anual de TB estimada en el 2001 en la Comunidad de Madrid fue de 20,9 casos por 100.000 habitantes; siendo la zona centro el área mas afectada con 40,7 casos por 100.000 habitantes $(8,9)$. En el área de salud correspondiente a nuestro estudio, la incidencia estimada fue 11,14 casos por 100.000 habitantes, cifra probablemente subestimada porque no todos los casos de TB son detectados en áreas especializadas.

Según datos de la Comunidad de Madrid, la curva de incidencia de TB sigue un patrón bimodal con dos picos. La incidencia más elevada se observa en el grupo de edad de mayores de 74 años, seguido por otro grupo con edades entre 25-34 años (8). En cambio en nuestro estudio, predominan los adultos jóvenes, por el impacto del VIH; y en segundo lugar figuran los ancianos, dada la importante comorbilidad y la instituonalización de este grupo de edad (10-12).

La epidemia del sida contribuyó al resurgimiento de la TB en los países desarrollados durante los años 80 y 90, alcanzando una incidencia del $41 \%$ de enfermos con TB y coinfección VIH (1). En los últimos años, la mayor eficacia del tratamiento antirretroviral ha disminuido dicha incidencia $(2,13)$. No obstante, en nuestro hospital, la coinfección por el VIH fue el factor de riesgo más frecuentemente observado (43\%), probablemente por existir una importante unidad dedicada al control y tratamiento de pacientes VIH positivos. Así, destaca en nuestro estudio que el $85 \%$ de los casos de TB y coinfección VIH eran adictos drogas por vía parenteral.

En cuanto a la inmigración, constituye uno de los factores de riesgo más importantes de TB. Así en países como EEUU, más del $50 \%$ de los casos de TB ocurren en la población inmigrante $(4,10)$. En España, aunque la inmigración ha aumentado considerablemente en los últimos años, sólo el $25 \%$ de los casos nuevos de TB aparecen en inmigrantes $(9,14)$. En nuestro estudio, sorprende que el porcentaje de casos de TB diagnosticados en inmigrantes es mínimo (9\%). Igualmente llama la atención, que la mayoría de nuestros inmigrantes diagnosticados de TB llevaban más de un año residiendo en nuestro país. Suponemos, que la infección o reactivación de la TB ocurrió en España, probablemente facilitada por las deficientes condiciones de habitabilidad (hacinamiento) $(14,15)$.

Entre los métodos de diagnóstico microbiológico, la tinción de BAAR es el más rápido, económico y fácil, pero el cultivo positivo para $M$. tuberculosis tiene la mayor sensibilidad $(90 \%)$ (16). En nuestro estudio, ambas pruebas fueron útiles para el diagnóstico, pero en los VIH positivos fue más rentable el cultivo que el BAAR.

El estudio de resistencia a los fármacos antituberculosos está indicado fundamentalmente en enfermos VIH, inmigrantes de países con resistencias primarias muy altas, formas clínicas muy graves (TB meníngea y miliar) y en pacientes que hayan recibido tratamientos antituberculosos previos (resistencias secundarias) $(9,15,17)$. A pesar de lo anterior en nuestra serie, sólo se realizó estudio de resistencias en el $30 \%$ de todos los casos diagnosticados de TB. 
La mayoría de nuestros pacientes no tenían antecedentes previos de TB ni de tratamiento tuberculostático, como en otros trabajos sobre el tema $(2,9)$. En nuestra experiencia, la localización más frecuente de las formas activas de TB fue la pulmonar, como en otros estudios revisados $(9,18,19)$. En cambio, no se observó ningún caso de TB miliar, que en otras series aparece en el 0,6\% (20).

Aunque las manifestaciones clínicas de TB a menudo son inespecíficas, en nuestra serie los síntomas respiratorios fueron los más frecuentes. En los pacientes con infección VIH, no es raro que incluso con TB activa la radiografía de tórax sea normal (21), como ocurrió en más de la mitad de nuestro casos coinfectados por VIH.

El tratamiento antituberculoso durante los dos primeros meses incluye habitualmente tres fármacos (isoniazida, rifampicina y pirazinamida). Solamente cuando las tasas de resistencia son mayores del 4\%, se debe añadir etambuntol o estreptomicina (3). A pesar de lo cual, en nuestra serie hasta un $37 \%$ recibieron etambutol durante los dos primeros meses. Cuando existe coinfección VIH, si se ha completado correctamente el tratamiento, la TB presenta bajo riesgo de recidiva $(<5 \%)$ durante el primer año (6). En nuestro estudio el incumplimiento terapéutico fue mayor entre la población VIH positiva, dada la frecuencia de drogadictos e indigentes (22).

\section{Bibliografía}

1. Iglesias MJ, Rabanaque MJ, Gómez LI. La Tuberculosis en la provincia de Zaragoza. Estimación mediante el método captura-recaptura. Rev Clin Esp 2002; 202 (5): 249- 254.

2. Campos F, Muñoz F, Umbría S et al. Evolución de la incidencia de la Tuberculosis en el área del sur de Sevilla en la década de los noventa. Arch Bronconeumol 2002; 38 (5): 214-220.

3. Small PM, Fujwara PI. Management of tuberculosis in the United States. N Engl J Med 2001; 345; 189- 199.

4. Geng E, Kreiswirth B, Driner C et al. Changes in the transmission of tuberculosis in the New York city from 1990 to 1999. N Eng J Med 2002; 346: 1453 - 1458 .

5. Lado FL, Barrio E, Carballo E, Carbacos A. Clinical presentation of tuberculosis and degree of inmunodefinciency in patients with HIV infection. Scand J Infect Dis 1999; 31: 387-391.

6. Lado FL, Prieto A, Cabarcos A et al. Recidiva de la tuberculosis en los pacientes infectados por el virus de la inmunodeficiencia humana. An Med Interna (Madrid) 2001; 18: 243-247.

7. Jasmer RM, Nahid P, Hopewell PC. Latent tuberculosis infection. N Eng J Med 2002; 347: 1860-1866.

8. Informe regional de casos de Tuberculosis de la Comunidad de Madrid. Informe del año 2001. Boletín epidemiológico de la Comunidad de Madrid 2003; 9 (9): 3-45

9. Grupo de trabajo del PMIT. Incidencia de la tuberculosis en España: resultados del proyecto multicéntrico de investigación en tuberculosis (PMIT). Med Clin (Barc) 2000; 114: 530-537.

10. Euro TB (CESES/KNCV) and the national coordinators for tuberculosis surveillance in the WHO European Region Surveillance of tuberculosis in Europe. Report on Tuberculosis in cases notified in 1996. Septiembre 1998.

11. Viloria A, Ribera JM. Criterios clínicos de sospecha de Tuberculosis en pacientes de edad avanzada. Rev Clin Esp 2002: 202: 388-390.

12. Lado FL, Tuñez V, Golpe AL, Cabarcos A, Pérez del Molino ML. Tuberculosis en pacientes ancianos. Formas de presentación. An Med Interna (Madrid) 2002; 1 (9): 111- 114.

13. Johnson JL, Okwera A, Hom DL et al. Duration and efficacy of treatment of latent tuberculosis infection in HIV - infected adults. AIDS 2001; 16: 2137-2147.

14. Díez Ruiz-Navarro M, Caloto González M. Grupo de trabajo del PMIT.
A pesar de disponer de un tratamiento efectivo, la TB sigue siendo una causa de muerte importante, en nuestros días. La mortalidad se atribuye a retrasos en el diagnóstico, a la coinfección VIH y otras patologías o la infección por cepas multirresistentes (23); en nuestra serie la mortalidad fue del $10 \%$, ligeramente superior a otros estudios revisados $(22,24)$. Sorprende que en nuestra población VIH la mortalidad fue mucho menor $(0,03 \%)$. Probablemente dicha mortalidad esté infravalorada por la dificultad de seguimiento de estos pacientes, pues según datos de los CDC, los pacientes VIH positivos presentan una mortalidad 4 veces superior a los no infectados por VIH (25).

En conclusión, la TB sigue vigente en nuestros días, con una incidencia mantenida, aunque puedan cambiar algunas de sus características. Los factores de riesgo más frecuentes en la población estudiada han sido la infección por VIH y la drogadicción (ADVP). Un factor de riesgo emergente, es la población inmigrante, colectivo difícil de manejar por su situación frecuentemente ilegal. Así como, los ancianos, especialmente los institucionalizados con enfermedades crónicas, donde es frecuente la reactivación de la enfermedad. Por todo ello, es necesario esforzarse en la detección precoz de la TB, con seguimiento y el control de los enfermos hasta conseguir la definitiva erradicación de la enfermedad.
Epidemiología de la Tuberculosis en minorías étnicas e inmigrantes en España. Prevención de VIH/SIDA en inmigrantes y minorías étnicas. Plan nacional sobre drogas. Ministerio de Interior. Madrid 2001.

15. Ramos JM, Masiá M, Rodríguez JC, Padilla I, Soler MJ, Gutiérrez F. Tuberculosis en inmigrantes: diferencias clínicoepidemiológicas con la población autóctona (1999-2002). Enferm Infecc Microbiol Clin 2004 22: 315-8.

16. Gómez E. Laboratorio de Microbiología y Tuberculosis. Rev Clin Esp 2002; 202: 16-19.

17. Grupo de estudio de Tuberculosis resistente en Madrid. Estudio transversal multihospitalario de Tuberculosis y resistencias en Madrid. Med Clin (Barc) 1996; 106: 1-6.

18. Reported Tuberculosis in United States 1999. Atlanta: Centers for disease control and prevention, August 2000.

19. Vieria Pascual MC, Bischofberger C, Pérez de Oteyza C. Estudio epidemiológico de los pacientes diagnosticados de Tuberculosis en el área noroeste de Madrid. An Med Interna (Madrid) 2003; 20: 1015 .

20. Inchaurraga I, Herrejón A, Plaza P, Blanquer R. Tuberculosis miliar, ganglionar, pancreática y costal. Presentación clínica y revisión bibliográfica. An Med Interna (Madrid) 2001; 18: 483-485.

21. Havlir DV, Barnes PF. Tuberculosis in patients with Human Immunodeficiency Virus Infection. N Eng J Med 1999; 340 (5): 367-373.

22. Caminal L, Trapiella L, Telenti M, Fernández Belnando de Quirós J. Características de la Tuberculosis en un hospital general durante los años 1993-1998. Análisis de las resistencias y coinfección VIH. Enferm Infecc Microbiol Clin 2002; 20 (2): 68-73.

23. Golpe Gómez R, Cifrián Martínez JM, Fernández Rozas SM, Fernández Infante B, Martín Martínez B, Alonso Martín MA. Características de los pacientes fallecidos por Tuberculosis. An Med Interna (Madrid) 1999; 16: 65-68.

24. García Ordóñez MA, Coimenero JD, Valencia A, Pérez Frías J, Sánchez González J, Orihuela F, et al. Incidencia y espectro clínico actual de la Tuberculosis en un área sanitaria metropolitana del sur de España. Med Clin (Barc) 1998; 110: 51-5.

25. Grupo de estudio de Tuberculosis resistente en Madrid. Estudio transversal multihospitalario de Tuberculosis y resistencias en Madrid. Med Clin (Barc) 1996; 106: 1-6. 